\title{
Partial Breast Reconstruction with Lateral Chest Wall Perforator Flap to Facilitate Breast Conservation in Breast Cancer: First 100 Cases with Cancer Outcomes at 8 Years Follow-Up and the Lessons Learned
}

\author{
Pankaj Gupta Roy' \\ Laura Mustata ${ }^{1,2}$ \\ Jesse $\mathrm{Hu}^{\mathrm{l}, 3}$ \\ Ben Phillips ${ }^{4}$ \\ Vaishali Parulekar ${ }^{5}$ \\ Madhu Bhattacharyya ${ }^{5}$ \\ Adrian Harris (D) $^{6}$ \\ Sileida Oliveros ${ }^{6}$ \\ 'Department of Breast Surgery, Oxford \\ University Hospitals NHSFT, The \\ Churchill Hospital, Oxford, UK; \\ ${ }^{2}$ Department of Obstetrics and \\ Gynecology, Filantropia Clinical Hospital, \\ Bucharest, Romania; ${ }^{3}$ Department of \\ General Surgery, National University \\ Health System, Singapore; ${ }^{4}$ Department \\ of Breast Pathology, Oxford University \\ Hospitals NHSFT, The Churchill Hospital, \\ Oxford, UK; ${ }^{5}$ Department of Breast \\ Radiology, Oxford University Hospitals \\ NHSFT, The Churchill Hospital, Oxford, \\ UK; ${ }^{6}$ Department of Breast Oncology, \\ Oxford University Hospitals NHSFT, The \\ Churchill Hospital, Oxford, UK
}

Correspondence: Pankaj Gupta Roy Department of Breast Surgery, Oxford University Hospitals NHSFT, The Churchill Hospital, Old Road, Oxford, OX3 7LF, UK

Tel +44 1865220932

Email pankaj.roy@ouh.nhs.uk
Background: This is a prospective cohort study of partial breast reconstruction (PBR) with a lateral chest wall perforator flap (LCWPF) to facilitate breast conservation surgery (BCS) for women undergoing surgery for breast cancer. The study was undertaken to study the clinical and cancer outcomes.

Methods: Patients diagnosed with ductal carcinoma in situ (DCIS) or breast cancer who consented to undergo BCS with PBR with LCWPF were included in the study. A prospective database has been maintained to collect information on clinico-pathological features, complications, and follow-up. Patients were asked to complete an anonymised PROM questionnaire over the years. The hospital electronic records were interrogated for women who have completed 5 years follow-up to assess for development of recurrence/events.

Results: A total of 105 patients underwent PBR with LCWPFs between 2011 and 2018. Of these, $74 \%$ underwent cancer resection and PBR as one operation whilst $26 \%$ underwent PBR as a twostage approach. The median tumor size on pre-op imaging was $30 \mathrm{~mm}$ for the one-stage approach and $39.5 \mathrm{~mm}$ for the two-stage approach ( $p$-value $=0.003$ ). The complication rates were low and the re-operation rate for close margins was $10 \%$, with $4 \%$ eventually requiring mastectomy. Good-toexcellent esthetic outcomes were reported in more than $80 \%$ of cases by patients and clinicians. The local recurrence rate (LR) was $2 \%$, distant recurrence rate $10.5 \%$, disease free survival (DFS) $86 \%$, distant disease-free survival (DDFS) $89 \%$ and overall survival (OS) $94.8 \%$ at 4.5 years median follow-up. This procedure provides an effective oncological approach, avoiding mastectomy with a good-to-excellent cosmetic outcome. The follow-up data establishes the safety of this approach. Discussion: This is the first published series of recurrence and survival data in patients undergoing PBR. We intend to continue with data collection to assess long-term outcomes beyond 10 years. The authors would recommend consideration of this technique to facilitate BCS and avoid mastectomy.

Registration: Not applicable.

Keywords: lateral chest wall perforator flap, breast conservation surgery, partial breast reconstruction, PBR, PROMs, breast cancer, breast cancer outcomes

\section{Introduction}

The surgical management of breast cancer has witnessed a considerable evolution in the past couple of decades, with emergence and wider adoption of oncoplastic 
breast surgery techniques, ${ }^{1}$ with emphasis on de-escalation of treatment where appropriate and discovery of new drugs. ${ }^{2}$ The aim is to facilitate breast conservation surgery (BCS) with emphasis on improved esthetic outcomes to improve quality-of-life and patient satisfaction. ${ }^{3,4}$

Oncoplastic breast-conserving techniques are classified as volume displacement or volume replacement. Autologous tissue is the preferred approach for volume replacement or partial breast reconstruction (PBR). The use of implants for volume replacement in PBR is hampered by problems with postoperative surveillance and by the need for radiotherapy, hence outcomes are generally poor. $^{5}$

Historically, PBR dates to 1986, with a published paper about the use of a random pattern local flap (lateral thoracodorsal artery flap) to assist implant based reconstruction after mastectomy for cases of breast cancer. ${ }^{6}$ Oncoplastic procedures have had an important development since CWPFs were first described; these flaps extend options for breast conservation with a positive psychosocial impact for the patients, the recovery is quicker, with low morbidity and high patient satisfaction with the esthetic outcome. ${ }^{7,8}$

The defects in the lower aspects of the breast can be addressed using local flaps such as abdominal adipofascial flaps ${ }^{9,10}$ or thoraco-epigastric perforator flaps along the inframammary fold based on anterior intercostal artery perforators and the superior epigastric artery. ${ }^{11-13}$

The defects in the lateral half of the breast can be reconstituted with lateral chest wall perforator flaps including the lateral intercostal artery perforator flap (LICAP), lateral thoracic artery perforator flap (LTAP), and thoracodorsal artery perforator flap (TDAP).,14-17 The distant flaps (LD, omental) ${ }^{18,19}$ used for volume replacement after partial reconstruction are most commonly pedicled, though some small case series of free flap volume replacement after partial mastectomy have been published. $^{20,21}$ This article focuses on PBR with lateral CWPF, commonly used perforators being LICAP and LTAP (Figure 1).

PBR with lateral CWPF is usually extended to small/ moderate sized non-ptotic breasts for laterally placed tumors. In selected cases, this procedure could be used for tumors with central or medial extent. ${ }^{22}$ PBR could be offered as a one-stage approach encompassing oncological resection $^{7}$ and PBR altogether or as a two-stage approach to ensure adequate oncological resection prior to $\mathrm{PBR} .{ }^{23}$
In this article, we present the results of a prospective cohort study on partial breast reconstruction using lateral CWPF in women undergoing breast conservation surgery for breast cancer assessing the oncological and esthetic outcomes over a long-term follow-up. To our knowledge, this is the first article reporting oncological outcomes over a medium-term follow-up for partial breast reconstruction facilitating breast conservation in breast cancer.

\section{Methods}

One hundred and five female patients diagnosed with invasive breast cancer or extensive in situ carcinoma of the breast, in a dedicated breast cancer unit in a University Hospital, were treated with breast conservation with partial breast reconstruction using lateral CWPF, over a period of 7 years. The patients included in the study were operated on by single surgeon between 2011-2018 and were followed up as per agreed hospital guidelines. The data was collected prospectively to study the clinico-pathological features, oncological and esthetic outcomes, patient reported outcomes, complications after surgery, and adjuvant treatment received. The demographic information and potential identifiable risks and comorbidities and treatment were collected from the hospital database. The database was updated regularly by collating from histological records, radiological reports, operation notes, clinic letters, and hospital episodes. The surgical team followed the patients to assess for clinical and esthetic outcomes, complications or significant events over 5 years after diagnosis. The hospital electronic patient records (EPR) were checked for patients beyond a 5-year follow-up period to check for development of events/recurrence and confirm compliance with the screening mammogram.

The outcomes studied were a) need for further breast surgery due to incomplete cancer excision, b) rate of complications after PBR, c) esthetic outcomes as assessed by the surgical team and the patients, and d) oncological outcomes to assess the safety of procedure including local recurrence (LR), distant recurrence (DR), breast cancer specific distant disease-free survival (DDFS), breast cancer specific disease-free survival (DFS), and overall survival (OS). The study was carried out as a part of routine clinical care with Oxford University Hospital ethics committee approval to study the outcomes. The ethical and clinical guidelines were adhered to and patients' permission was obtained to use their anonymized photographs for educational and publication purposes. This study was conducted in accordance with the Declaration of Helsinki. 


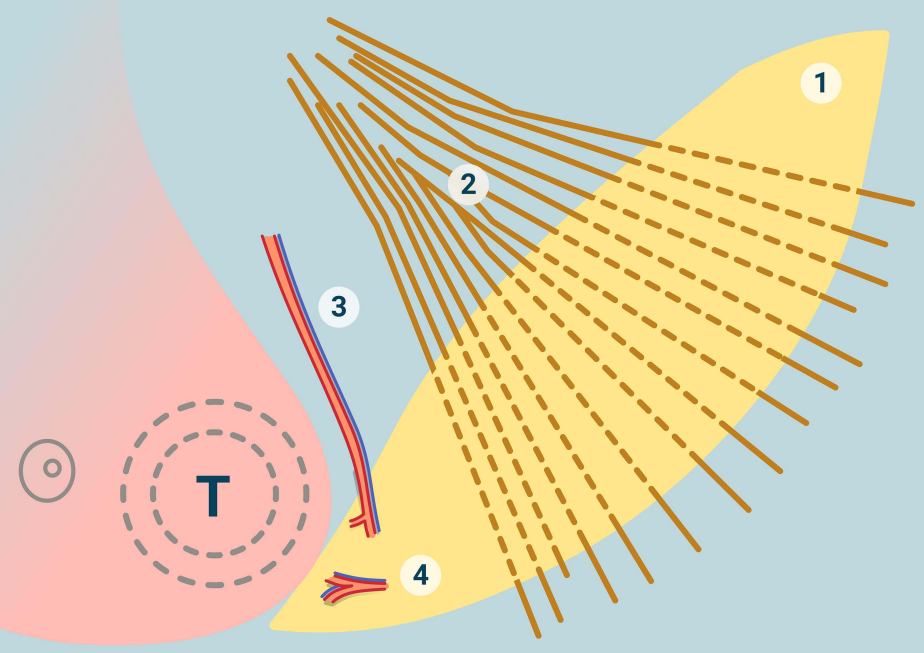

Figure I Schematic representation of the anatomy of lateral chest wall perforator flaps (CWPF) and the blood supply in relation to breast. T: tumour with outer circle representing the wide local excision; I) lateral CWPF; 2) latissimus dorsi muscle; 3) lateral thoracic vessels; 4) lateral Intercostal vessels.

The questionnaire used to assess the patient reported outcomes were Breast Q and BIS (Breast Image score); both have been validated for use in women undergoing surgery for breast cancer. The anonymized questionnaires were sent out by a member of the surgical team between 4-6 months after completion of radiotherapy. BIS was used in the earlier years, as that was the widely used tool available; however with development of more specific tool for breast conservation surgery, the team switched to Breast Q. ${ }^{24}$ The clinical team also assessed the esthetic outcomes over the years. Two surgeons (one trainee and one senior surgeon) reviewed preoperative and post-op photographs at 12 months for each patient and the esthetic outcomes were marked subjectively using Harris scale (poor, fair, good, or excellent). The clinical photographs at 1-year post surgery and between 3-5 years follow-up were reviewed and compared to assess for stability of the esthetic result and potential impact of radiotherapy over time.

\section{Statistical}

The data were statistically described in terms of mean median and range, or frequencies (number of cases) and percentages when appropriate. Comparison of numerical variables (tumor size) between the study groups was done using unpaired 2-tailed Student $t$-test (alpha $=0.05$ ).

\section{Patient Selection}

The patients included in our study were diagnosed with breast cancer or ductal carcinoma in situ (DCIS) between 2011 and 2018. All patients were discussed in the hospital breast cancer multidisciplinary team meeting and management was guided as per national guidelines. The surgical indication took into consideration the size, site, and type of tumor, size of associated DCIS, multifocality, breast size, patient preference, and tumor/breast size ratio.

The imaging investigations performed included mammogram and ultrasound of breast and axilla followed by biopsy. MRI of breasts was carried out for selected cases 
including lobular histology, discrepancy in sizing the tumor, for all patients undergoing neoadjuvant chemotherapy and women with dense breasts. The whole-body scans to stage the cancer was requested where appropriate. The surgical choices including the option for BCS and PBR with lateral CWPF were discussed with the patient in the presence of a breast care nurse. Patients were provided with the information leaflets on their options and given adequate time to make an informed decision.

\section{Patient and Public Involvement}

The study was driven by the need to prove the safety of breast conservation approaches being undertaken within the umbrella of oncoplastic surgery. Partial breast reconstruction with lateral CWPF is not yet a commonly practiced technique and the published data on the oncological outcomes is very limited. There is evidence to suggest the improved esthetic outcomes, therefore acceptability of the approach by patients. All patients included in this study were informed about and consented to the participation as per local hospital ethics committee guidelines.

\section{Results}

One hundred and five female patients diagnosed with breast cancer or DCIS with an age range of 30-78 years (mean age was 52 years) were treated with BCS and PBR with lateral CWPF (Table 1).

\section{Investigations}

All patients had bilateral mammograms, ultrasound, and biopsy to confirm diagnosis. Thirty-four patients had MRI to either size the tumor or assess for multifocality. Ten patients were active smokers at presentation. Thirty-seven patients were screen detected and 68 presented symptomatically. The majority showed invasive ductal cancer on histology, 16 were lobular carcinoma, two cases were of mixed type, and eight cases had DCIS only. Fifty-seven out of 80 invasive cancers that underwent primary surgery $(71 \%)$ had DCIS associated with the invasive cancer. Sixteen (16.4\%) patients had HER-2 positive disease, 18 had triple negative breast cancer (18.5\%), whilst the majority were ER positive (Luminal subtype).

\section{Type of Surgery}

All patients included in the study had BCS, and the ones with proven invasive cancer had SLNB for axillary staging. One patient had HER-2 positive metastatic disease at presentation and underwent surgery for in-breast disease progression but stable distant disease and the surgery was limited to the breast (no axillary surgery performed).

Most patients were offered PBR at their initial surgery. Four patients were offered partial breast reconstruction during margin re-excision as the pathological tumor size was significantly more than that anticipated at the initial imaging.

\section{Breast and Tumor Size}

The breast sizes vary from AA cup to D cup; the distribution is shown in Table 2. The mean tumor size was significantly bigger in women with $\mathrm{C} / \mathrm{D}$ cup $(35.6 \mathrm{~mm})$ compared to women in this series with $\mathrm{AA} / \mathrm{A} / \mathrm{B}$ cup $(29.6 \mathrm{~mm})$ ( $p$-value $=0.03$, student $t$ unpaired test).

The tumor sizes are tabulated in Table 1 . The majority of the invasive cancers undergoing primary surgery were T2 (62\%), 34\% were T1 (majority T1c), and a small minority were T3. Although the invasive cancer dictates T staging, the tumor resection volume is dictated by associated DCIS and multifocality. Almost a quarter of invasive cancers in this series needed much larger volumes of tissue excised than suggested by the $\mathrm{T}$ status; 14 had bifocal or multifocal tumor and 10 had DCIS extending well beyond the invasive cancer to warrant a substantial $(>50 \%)$ increase in volume of tissue excised. Ninety percent of tumors were in outer quadrants; a small proportion (10\%) were in other quadrants.

Preoperatively, the tumor size on imaging varied between 15 and $100 \mathrm{~mm}$ (median $30 \mathrm{~mm}$ ). The majority (78) had PBR with CWPF along with BCS as a one-stage procedure (74\%) (Figure 2); 27 patients (26\%) had surgery as a two-stage approach. Overall, multifocality was diagnosed in $14(15 \%)$ patients.

The 2-stage approach was adopted for women (27 patients) with a high tumor-to-breast ratio, thus bordering on to recommendation for mastectomy. The mean size on pre-op imaging was $39.9 \mathrm{~mm}$ (ranging from $18-75 \mathrm{~mm}$ ); this was significantly higher than the mean tumor size observed in the one-stage group $(p=0.003$, unpaired Student's $t$-test $)$ The tumor characteristics for patients undergoing two different approaches are detailed in Table 3. The two groups were similar with regards to distribution of node positive, Her-2 positive, and triple negative cancers.

\section{Axillary Nodal Disease}

Out of 96 invasive cancers (excluding one metastatic cancer), 15 patients $(15.6 \%)$ had positive nodal disease at presentation (Figure 3). Patients with node negative disease underwent SLNB and 49 (51\%) were node negative. 
Table I Distribution of the Clinic-Pathological and Treatment Parameters

\begin{tabular}{|c|c|}
\hline Tumor Characteristics and Treatment & Number of Patients $(\%)($ Total = I05) \\
\hline Symptomatic presentation & $76(72.4 \%)$ \\
\hline Screen detected cancers & $29(27.6 \%)$ \\
\hline Invasive cancers & 97 (I metastatic at presentation) $(92.4 \%)$ \\
\hline IDC & $78(80 \%)$ \\
\hline ILC & $14(14.5 \%)$ \\
\hline Mixed & 3 \\
\hline Others (medullary, squamous) & 2 \\
\hline DCIS only & 8 (I had microinvasion) (7.6\%) \\
\hline High grade & 5 \\
\hline Intermediate grade & 3 \\
\hline Invasive tumor size on histology** (excluding NAC) & 80 \\
\hline $\mathrm{TI}$ & 27 \\
\hline $\mathrm{T} 2$ & 50 \\
\hline $\mathrm{T} 3$ & 3 \\
\hline Invasive tumor size on histology post-NAC & 16 \\
\hline $\mathrm{PCR}$ & 4 \\
\hline TI & 5 \\
\hline $\mathrm{T} 2$ & 6 ( 2 were bifocal) \\
\hline T3 & 1 \\
\hline \multicolumn{2}{|l|}{ *Tumor grade (invasive cancer only) } \\
\hline Gr I & 14 (15\%) \\
\hline Gr 2 & $42(43 \%)$ \\
\hline Gr 3 & $4 \mathrm{I}(42 \%)$ \\
\hline *Node positive at diagnosis & $15(15.6 \%)$ \\
\hline *Axillary nodes positive (total) & $47(49 \%)$ \\
\hline *Triple negative cancers & I8 (I8.5\%) (6 had NAC, 2 declined adjuvant chemo, 10 had adjuvant) \\
\hline *ER positive (invasive cancers) & $73(75 \%)$ \\
\hline *ER positive and Her-2 negative & $63(65 \%)$ \\
\hline *Her-2 positive cancers & 16 (I6.4\%) (ER+: I0, ER-: 6) \\
\hline *Neo-adjuvant chemotherapy (NAC) & $16(16.6 \%)$ \\
\hline *Patients received chemotherapy (adjuvant and NAC) & 57 (+3 declined) \\
\hline Patients received adjuvant radiotherapy & I00 (I declined, 4 were not recommended) \\
\hline *Patients received adjuvant endocrine therapy & 74 \\
\hline
\end{tabular}

Notes: * This information applies to invasive cancers only. ** Fourteen had multifocality and 10 had extensive DCIS to increase the whole tumor size by more than $50 \%$ (influencing the need for wider resection than indicated by $T$ status).

Abbreviations: NAC, neoadjuvant chemotherapy; pCR, pathological complete response.

Thirty-two had positive nodes with eight micrometastasis. Twenty-five were macrometastases; two consented to participate in POSNOC trial, ${ }^{25} 12$ had axillary node clearance, whilst 10 opted for nodal radiotherapy.

\section{Surgical Details}

All patients were operated on in lateral position to undertake PBR with CWPF. Handheld Doppler was used preoperatively to mark the perforators. The radiologist localized all impalpable tumors on the morning of surgery using wire and the ones that required wider resections amounting to quadrantectomy were localized with two or more wires. During surgery, the tumor excision was undertaken first to ensure there was no compromise to oncological resection. The median weight of the specimens excised was $95 \mathrm{~g}(20-220 \mathrm{~g})$.

Two perforators were dissected during surgery and the decision was made to keep one or both depending on the ease of mobility of the flap and assessment of flap vascularity. The perforators used included the LICAP, LTAP, and serratus branch of thoracodorsal (in a few cases). TDAP flap reconstructions have been excluded from this series as the authors believe that TDAP flaps require a higher level of skill and should not be equated with other chest wall perforator flaps.

Forty-four cases were based on LICAP, 33 were based purely on LTAP, 26 had both LTAP and LICAP, and two 
Table 2 Details of the Median Tumor Size in Relation to the Bra Cup

\begin{tabular}{|l|l|l|}
\hline Breast Size & $\begin{array}{l}\text { Number of } \\
\text { Patients }\end{array}$ & $\begin{array}{l}\text { Median Tumor Size on } \\
\text { Imaging (Pre-Op)* }\end{array}$ \\
\hline AA & 3 & 18 \\
\hline A & 7 & 24 \\
\hline B & 33 & 26 \\
\hline C & 39 & 33 \\
\hline D & 23 & 35 \\
\hline
\end{tabular}

Note: *Including patients who had NAC.

were based on the serratus branch of thoracodorsal vessels. The average operating time varied from 90 minutes to 3 hours (excluding axillary node clearances) depending on the size of the flap required and complexity of the wide local excision. The operating time reduced over time with experience gained by the surgical team.

Most patients underwent surgery as an overnight stay in the hospital. A drain was inserted during the surgery and removed the following day before discharge from the hospital. The post-operative pain relief was managed with simple analgesics with breakthrough pain relief by codeine. Patients were advised regarding post-op shoulder exercises to ensure full recovery within 2-3 weeks. Few patients needed dedicated physiotherapy input due to shoulder stiffness, particularly after radiotherapy.

\section{Histology}

The median pathological tumor size was $27 \mathrm{~mm}$ (12$140 \mathrm{~mm}$ ) (excluding neoadjuvant chemotherapy). The median distance of radial excision margins was $10 \mathrm{~mm}$ (2$15 \mathrm{~mm}$ ) in adequately excised tumors (the acceptable radial margin was $2 \mathrm{~mm}$ as per hospital policy). Of 78 patients undergoing PBR as a one-stage approach, six showed inadequate excision at one margin and two patients had inadequate excision of multiple margins; all eight (10.2\%) required further surgery. Five underwent successful margin re-excision and three were recommended mastectomy due to either extensive DCIS (two cases) or multifocal unexpected

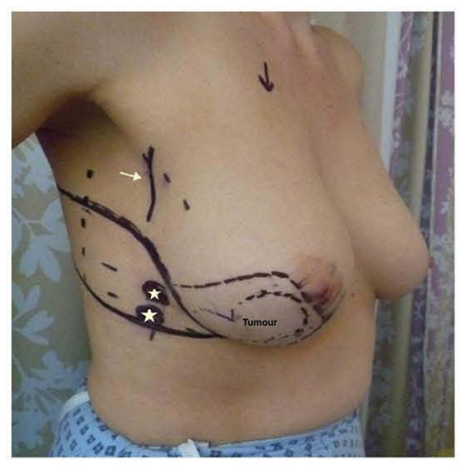

A

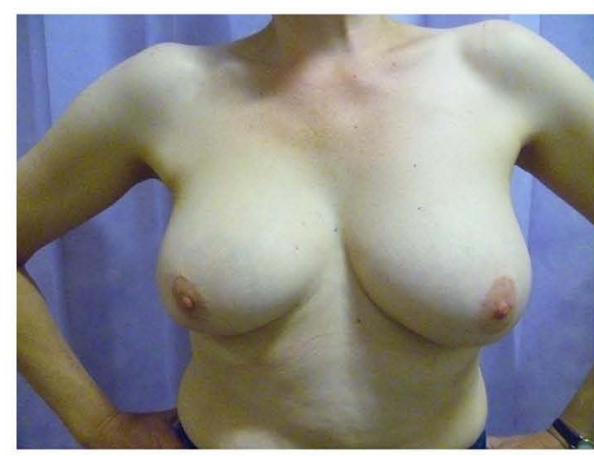

D

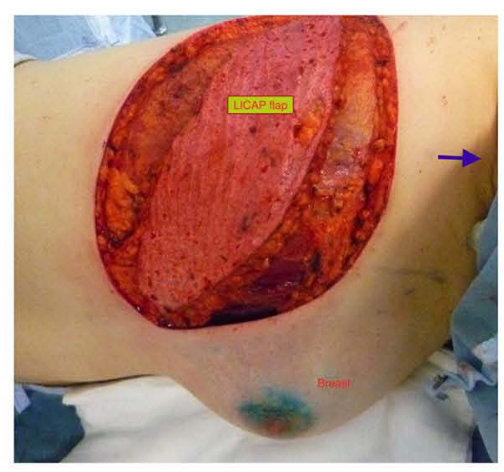

B

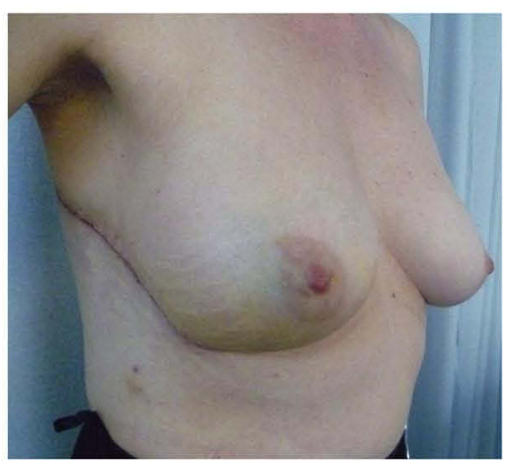

C

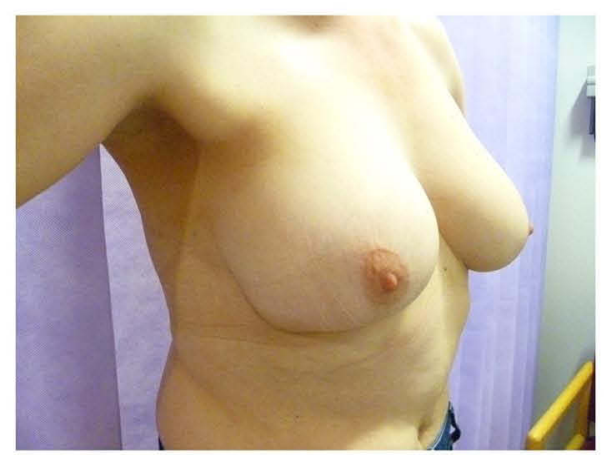

$E$

Figure 2 One-stage LICAP flap reconstruction (A) 43-year old with $40 \mathrm{~mm}$ cancer in the lower outer quadrant of the right breast. Pre-op marking for tumor location and lateral CWPF. The solid vertical line (white arrow) denotes surface marking for lateral thoracic artery (LTA) and stars represent lateral intercostal artery perforators (LICAP). The dotted lines are along the lateral border of pectoralis major and anterior border of latissimus dorsi muscle. (B) Intra-operative picture showing the flap dissected (arrow points towards head with patient in lateral position). (C) 4 weeks post-op with scar on the lateral chest wall. Patient had chemotherapy after surgery. (D) Appearance and symmetry of breasts 4 years after radiotherapy. (E) Appearance of scar 4 years later. 
Table 3 Tumor Characteristic of Patients Undergoing One-Stage and Two-Stage Approaches

\begin{tabular}{|l|l|l|l|}
\hline & One-Stage (78) & Two-Stage (27) & $p$-value \\
\hline Mean tumor size (mm) (on pre-op imaging) & 30.7 & 39.9 & 0.003 \\
Total number of patients & 78 & 27 & \\
DCIS only & 4 & 4 (I with microinvasion) \\
Invasive cancers & $74^{\$}$ & 23 & 5 \\
Neo-adjuvant chemotherapy & 11 & $14(64 \%)$ & \\
Chemotherapy (adjuvant and NAC) & $46^{*}(61 \%)$ & $4(18 \%)$ & \\
Her-2 positive cancers & $12(16 \%)$ & $12(54 \%)$ & \\
Node positive cancers & $35(47 \%)$ & $3(14 \%)$ & \\
TNBC & $15(18 \%)$ & \\
\hline
\end{tabular}

Notes: * Three declined. ${ }^{\$}$ One patient had metastatic cancer.

ILC (one case). Two patients underwent completion mastectomy with direct to implant reconstruction, whilst one declined further treatment.

Eight patients were treated for DCIS only and 97 patients had invasive disease.

\section{Adjuvant Therapy}

Sixty out of 97 (61\%) patients with invasive cancer were recommended chemotherapy; three patients declined. Of 57 patients, 16 underwent neoadjuvant chemotherapy (NAC) and 41 had adjuvant chemotherapy. Twenty-five percent (4/16) had pathological complete response (pCR) after NAC. The tumor size distribution after NAC is shown in Table 1, with the majority showing partial response to therapy.

Ninety-five percent (100 patients) underwent adjuvant radiotherapy (RT). Two had completion mastectomy so were not recommended for radiotherapy. One patient declined further treatment despite inadequate surgery for extensive DCIS. Two patients were not recommended RT due to low benefit. Twenty-six patients also received RT to regional nodes. The most common RT fractionation used was 50 Gy in 25 fractions. However, clinicians felt more confident to apply results of Start B trial and gradually started using 40 Gy in 15 fractions after oncoplastic breast conservation surgery from 2017. Tumor bed boost was offered to $59 \%$ of the patients on the basis of age (under 50 years old), grade 3 , tumor size $>3 \mathrm{~cm}$, margins, and other high-risk factors. From those patients receiving a tumor bed boost, 38\% had an electron boost, and $61 \%$ required a photon boost delivered using mini-tangential fields.

Seventy-four patients were recommended hormonal therapy, as appropriate, according to their menopausal status. As per our guidelines, DCIS patients were not routinely recommended hormone therapy until 2018. Only one patient with DCIS elected for tamoxifen as she presented with bilateral DCIS and decided against breast radiotherapy.

\section{Complications}

The complications encountered in this cohort of patients include:

Post-op complications were seen in 20 patients (19\%) in our study.

1. Immediate re-operation for complications (three patients)

a. Hematoma (two patients): Both patients returned to theater for evacuation and washout. One patient had a delay in return to theater, developed venous congestion of the flap requiring partial flap resection resulting in volume loss resulting in a suboptimal esthetic outcome.

b. Nerve entrapment presenting as post-op pain and inflammatory edema (one): diagnosed on wound exploration.

2. Superficial skin necrosis on the breast (one): managed conservatively but resulted in significant scarring with suboptimal esthetic outcome.

3. Minor complications (seven)

a. Small hematoma (one).

b. Seroma (two).

c. Stitch abscess/extrusion/minor wound dehiscence (four).

Conservative management was pursued with aspiration and/or antibiotics, with no significant impact on the further therapy or esthetic outcome. 


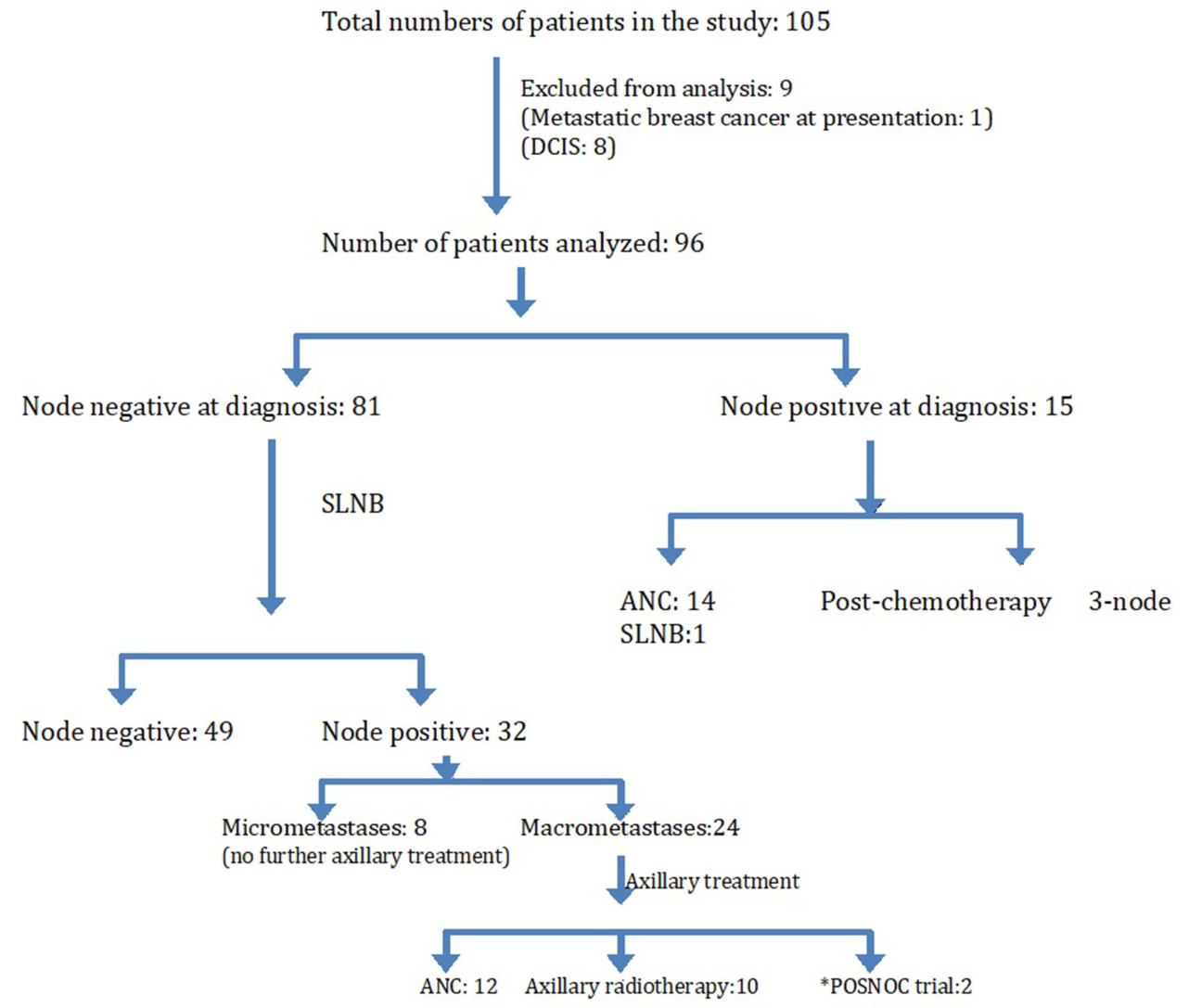

*POSNOC trial: RCT comparing standard axillary treatment to no further axillary treatment in breast cancer patients receiving primary surgical treatment with 1-2 positive nodes on SLNB.

Figure 3 Flowchart depicting axillary lymph node status along with axillary treatment.

1. Symptomatic Fat necrosis (four): diagnosed on ultrasound scan with confirmation on biopsy in two cases.

2. Nerve entrapment presenting as pain (five): It's not uncommon for patients to present with chronic pain of varying severity after breast surgery. Detailed assessment is essential including examination for trigger points, which are often along the scar suggesting sensory nerve entrapment. Most of these patients were treated with local steroid injection.

3. There was no flap loss encountered in the study.

\section{Medium Term Complication}

Breast lymphoedema (six patients): Two patients had an extensive area subjected to tumor-bed boost during radiotherapy resulting in radiotherapy injury and breast dema that took almost 2 years to settle. This was seen during earlier years of our experience; we adjusted the intra-operative method of marking the tumor bed after discussion with the radiation oncologist to avoid confusion with regards to the volume of tissue excised versus tumor bed. All patients were referred to the lymphedema clinic for expert advice and management.

\section{Pre-Existing Risk Factors}

Ten patients in the study were active smokers. Eight had one-stage PBR and two had a staged approach to PBR. All hada breast cup size between $\mathrm{C} / \mathrm{D}$ and the median tumor size on imaging was $35 \mathrm{~mm}(24-70 \mathrm{~mm})$. Two patients were obese with a BMI $>38$ and two patients had type 2 diabetics. Two post-op complications were observed in these patients (both smokers); one developed a patch of necrotic skin overlying the tumor excision and another developed nerve entrapment requiring wound exploration.

\section{Follow-Up and Oncological Outcomes}

All patients were followed up as per guidelines with an annual mammogram for at least 5 years after diagnosis or 
until the age of 50 years, whichever was longer (Figure 4). Of 105 patients, seven were lost to follow-up. The median follow-up period was 54 months (4.5 years) with a range of 27-103 months (2.3-8.5 years). Forty percent of the patients completed the mandatory 5-year follow-up and continued to receive 3-yearly National Breast Screening mammograms.

The presence of a flap did not interfere with the standard mammographic assessment (published elsewhere). ${ }^{26}$ We have already published that the recall and biopsy rates after PBR were similar to that observed after standard breast conserving surgery. ${ }^{27}$

Ten patients developed distant metastasis whilst two developed local recurrence without distant disease and one patient developed contralateral breast cancer in the absence of local or distant disease. Of the patients presenting with distant disease, seven relapsed with visceral metastasis within 15-40 months after diagnosis of triple negative breast cancer and five patients succumbed to the disease after distant relapse. One patient relapsed in liver and bones 2 years after initial diagnosis of Her-2 positive and two developed distant disease after treatment for ER positive and Her-2 negative cancer.

The patient with local relapse was diagnosed with a small single nodule of local recurrence in the areolar skin 2 years after initial treatment for T2N1, grade 2, ER positive Her-2 negative cancer and was on tamoxifen.
Regional and/or distant disease was ruled out and she underwent surgery for local recurrence. The other patient developed ipsilateral recurrent DCIS 7 years after the initial treatment having declined further surgery and/or radiotherapy after inadequately excised DCIS at initial presentation. She agreed to undergo completion mastectomy with immediate implant reconstruction.

One patient developed contralateral breast cancer 4.5 years after initial diagnosis of node positive lobular cancer. She needed a mastectomy and requested for bilateral mastectomies (BRCA mutation negative).

All patients were followed up in the surgical clinic for 5 years to assess for delayed complications and potential impact on esthetic outcomes over the years. The majority of the patients maintained esthetic outcomes for volume and symmetry over the 5-year follow-up, suggesting that the flap withstands radiotherapy well. Tw patients developed breast asymmetry within 2 years due to the combined effect of over-correction of the defect with CWPF and post-radiotherapy breast lymphedema. They underwent unplanned contralateral breast augmentation with implant to achieve symmetry (Figure 5).

\section{Esthetic Outcomes}

Separate audits were performed to assess patient satisfaction with the outcome. Quality-of-life questionnaires (Breast-Q

Total number of the patients recruited between 2011 and 2018: 105

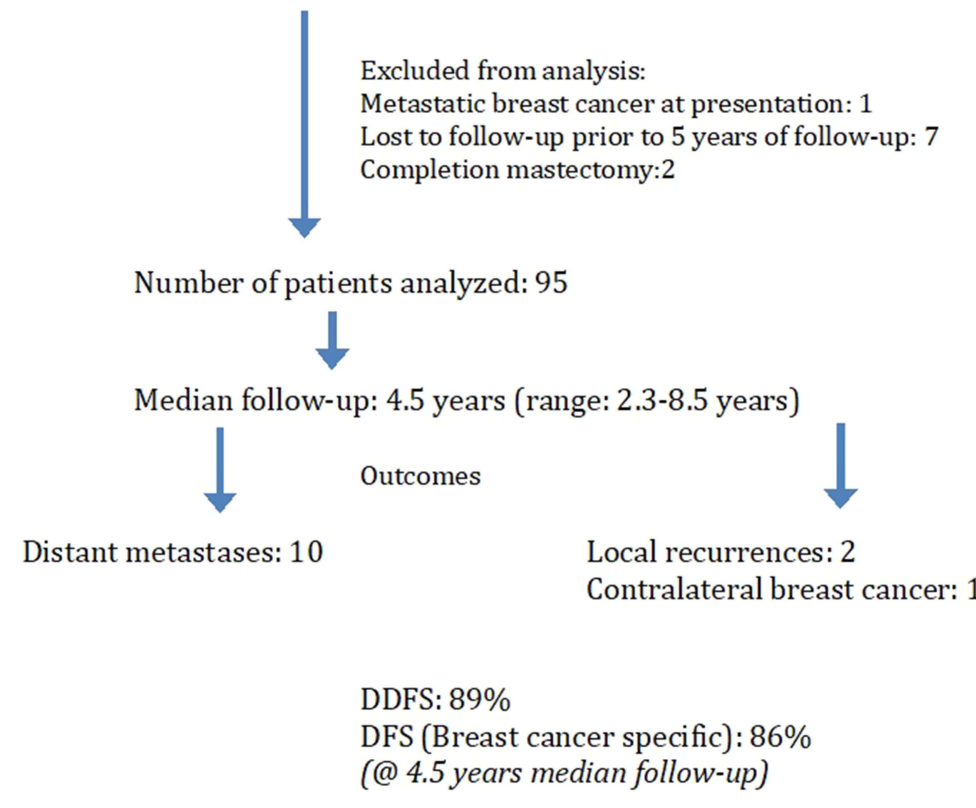

Figure 4 Cancer outcomes in the study (Consort format). 
and Body Image scale) ${ }^{28,29}$ were sent to patients with a $70 \%$ response rate. The questionnaires assessed for pre- and postoperative quality-of-life, sexual life, body image, functional impact on the arm and shoulder movement, neck pain or swelling, in addition to satisfaction with the medical team. Eighty per cent of responders reported high satisfaction with the esthetic outcome and $95 \%$ were satisfied with the treating team. Of the quality-of-life domains using Breast-Q, physical well-being was scored at $75 \%$, psychosocial wellbeing more than $80 \%$, whilst sexual well-being was at $60 \%$ (data presented as abstract at UK International Breast Cancer Symposium, 2018). However, the sexual well-being scores did not change significantly when compared with pre-op scores. With regards to physical discomfort, including neck pain, back pain, or swelling of the breast/arm, 73\% reported high satisfaction scores and $80 \%$ expressed satisfaction with back scar and shoulder function.

The esthetic outcome judged by the surgical team (Harris scale) has been good to excellent in over $80 \%$ of patients on assessing the clinical photographs (available for 91 patients). Three patients had sub-optimal results. Eleven patients were recorded as fair results due to breast asymmetry, nipple deviation, or flattening of contour. The authors observed maintained esthetic outcome with time in the majority of patients (Figure 6).

We did not observe any functional limitation of the donor site amongst the patients apart from self-limiting scar tightness and stiffness in the immediate post-op period.

\section{Discussion}

Partial breast reconstruction with chest wall perforator flaps is a significant addition to the oncoplastic breast surgery repertoire that could be offered to women to facilitate BCS without compromising cancer resection and outcomes and improved functional and aesthetic outcomes.

The majority (41\%) of flaps were based on LICAP vessels in our series. We would like to emphasize that there is a significant role of LTAP vessels in practicing this technique, as proposed by McCulley et al. ${ }^{17}$ Lateral thoracic artery/vessels (LTA) are present in about $80 \%$ of individuals and lend themselves to easy detection. In our series, $31 \%$ of flaps were raised purely on LTAP and an additional $26 \%$ of flaps had LTAP, which was the dominant vessel along with LICAP. The orientation and length of these vessels lend greater versatility in mobility and rotation of the flap, allowing better fill. Therefore, the authors have a preference for these vessels for bigger defects. However these vessels are prone to injury during axillary surgery, so caution needs to be exercised.

The data presented in this series confirms the safety of this approach with DDFS and DFS figures well within an acceptable range. ${ }^{30,31}$ To our knowledge, this is the first case series with medium-term oncological outcomes after partial breast reconstruction with lateral chest wall perforator flaps, although short-term outcomes have been reported. ${ }^{32}$ It is important to emphasize that $>50 \%$ of patients in this series had either node positive, triple negative, or Her-2 positive cancers (subset forming aggressive cancers) and median follow-up of 4.5 years with very low local recurrence confirms the suitability of BCS in women with a relatively larger tumor size. The breast conservation imparts a significant advantage with regards to improved psychosocial well-being in comparison with mastectomy with/without reconstruction. ${ }^{33}$ The absence of scarring on the breast in women undergoing one stage PBR, the unlikely need for contralateral symmetry surgery, ${ }^{34}$ quicker

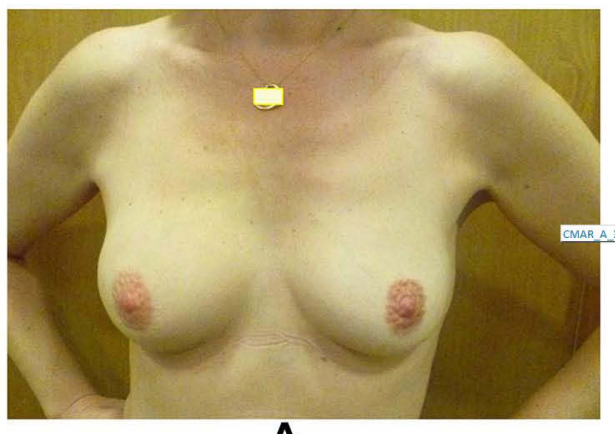

A

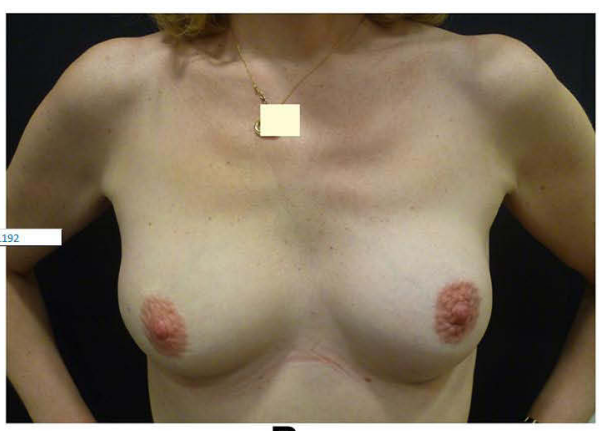

B

Figure 5 Patient before (A) and after (B) contralateral breast augmentation to address asymmetry after PBR with CWPF. The augmentation was performed 2 years after completion of cancer treatment. 


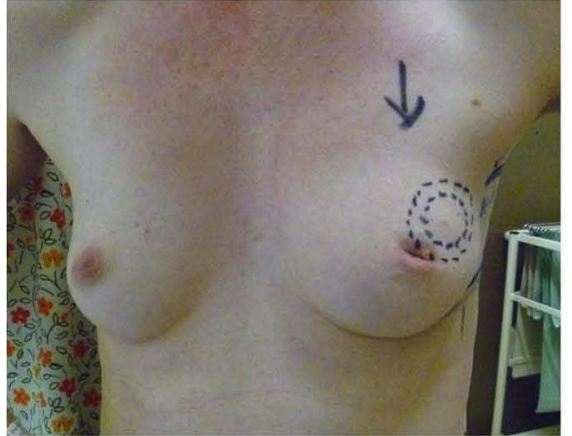

A

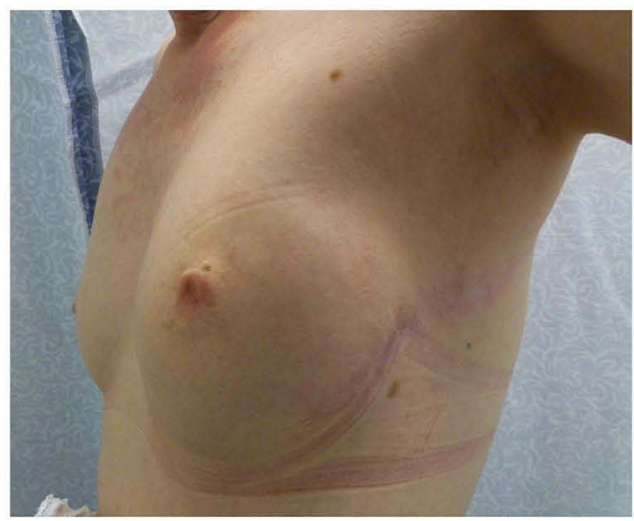

D

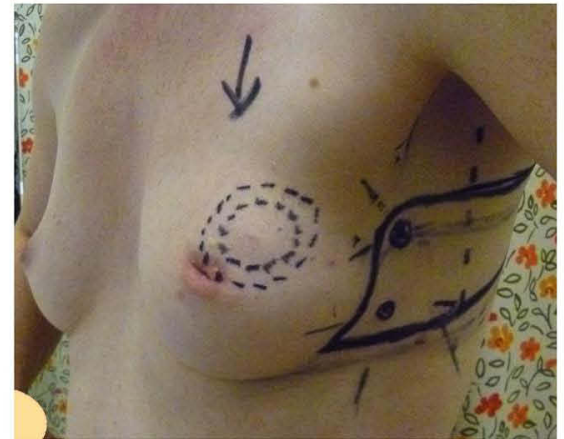

B

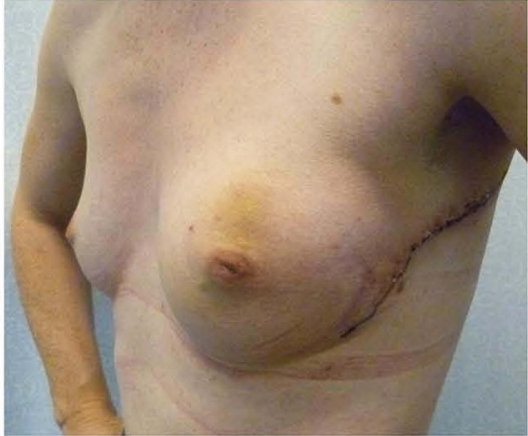

C

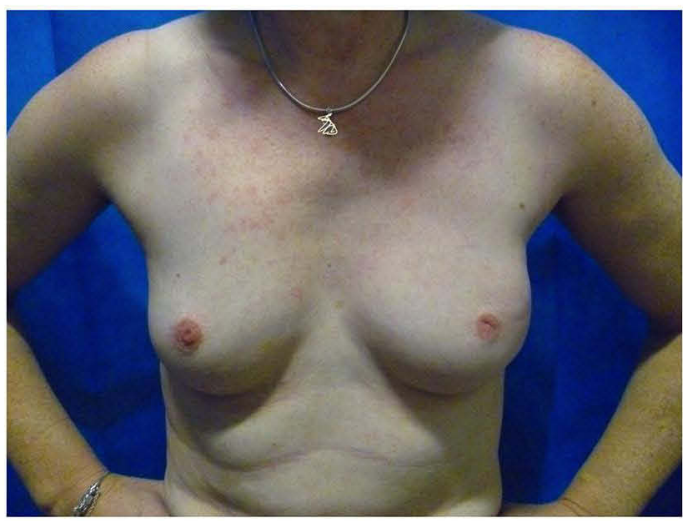

$\mathbf{E}$

Figure 6 Pre-op and post-op photographs of PBR with lateral CWPF in a slim patient with very small breasts. 42 -year old with $20 \mathrm{~mm}$ cancer in the upper outer quadrant of left breast with an "A" cup. (A) Pre-operative photograph. (B) Pre-op marking for CWPF. (C) 2 weeks after surgery. (D) I year after treatment. (E) 3 years after radiotherapy on right side. Patient had chemotherapy after surgery.

recovery, and short hospital stay in comparison with mastectomy and breast reconstruction are added benefits.

The authors observed maintained esthetic outcomes with time in the majority of the patients, suggesting that the flaps withstand radiotherapy well and shrinkage with time may be less of an issue with this approach. However, this may well reflect improvement in radiotherapy techniques and further follow-up would provide information with regards to long-term esthetic outcome.

We tabulate below the lessons learned with our experience over the past decade:

1. Intra-operative marking of tumor bed: It is not uncommon that wider excisions are performed either intentionally (for margins) or to create a tunnel laterally for flap inset. It is important that the marking clips are restricted to the tumor bed (and not to the entire surgical bed) as that could pose a challenge to the radiation oncologist during tumor bed boost localization. This is particularly important when relatively large invasive cancers are being excised to avoid boost being delivered to an even larger area, increasing the long-term risk of fibrosis and impaired cosmesis. ${ }^{35}$

2. Issues with sensory nerve entrapment: About $5 \%$ of patients required steroid injection and one patient required wound exploration to manage this complication in our series. The symptoms tend to vary from moderate to severe pain. Earlier, we made effort to preserve the sensory innervation to the flap but we have changed our practice to "not to" preserve the visible sensory nerve to the flap.

3. Choice between one stage and two-stage approach: The split between the two approaches is about $75 / 25$ in our practice, respectively. Of the patients selected for staged approach, most underwent mastectomy with or without reconstruction if the initial attempt at BCS was unsuccessful; however, four patients had only one close/involved margin, therefore a decision was made to proceed with PBR along with margin re-excision during the second operation, with success. We would recommend a limiting staged approach to those with a high tumor to breast size 
ratio due to the cost implications, logistics in theater planning, and subjecting patients through two hospital admissions. The two-stage approach is relevant for lobular cancers, DCIS, bifocal cancers, and post neoadjuvant chemotherapy, where pre-operative disease estimation could be challenging. ${ }^{23}$ The re-excision rate for the onestage approach observed in our series was low enough to advocate our practice. We did not observe any significant difference between the two groups with regards to complications, esthetic outcome, or patient satisfaction rates.

4. Extension of indications for women with very small breasts: Breast surgeons often face the limitations in surgical choices to offer to women with very small breasts (A/AA cup). These women are more likely to undergo mastectomy for small (even T1c) tumors or to succumb to poor esthetic outcomes. We have now extended the choice of PBR to carefully selected women with slim physique and small breasts provided there is lax tissue available on the lateral chest wall. These patients require small volumes for replacement proportionate to their body habitus and have done well in our series with a PBR approach.

5. Choice of perforators: The authors believe that TDAP flaps require a higher level of skills and training and should not be equated with other chest wall perforator flaps (LICAP and LTAP). Moreover, TDAP flap interferes with the ability to perform LD flap reconstruction in future so these flaps should be reserved for selected patients.

6. High risk patients: Patients with high BMI, active smokers, or diabetics are high risk patients, prone to complications and wound/flap problems. In our experience, CWPF allows BCS with lesser risk of wound complications in this "higher risk" group in comparison with other options, such as wise pattern therapeutic mammaplasty or mastectomy with immediate breast reconstruction. The perforator flaps are sturdy and resilient provided a good perforator is identified intra-operatively. There were 10 active smokers (two patients with BMI greater than 28 and two type 2 diabetics) in our study; we did not observe a higher complication rate in these patients. This extension of indication for CWPF should, however, be practiced with caution in clinical practice with sound clinical judgment by experienced surgeons and shared decision-making with patients.

We have published that the surveillance mammographic follow-up after PBR with CWPF in women undergoing BCS for breast cancer is accurate with low recall
$(2.2 \%)$ biopsy rates and comparable with routine BCS recall rates. $^{27}$

Our series reports a low complication rate in line with the published evidence. ${ }^{36}$ Less than $5 \%$ returned to theater for post-op complications; three immediate postoperatively and two for fat necrosis. Breast lymphoedema and breast pain due to trigger points were the main concerns reported in the medium-term follow-up. Four (4\%) patients developed clinically significant fat necrosis, which is well within the acceptable risk after breast surgery. ${ }^{37}$

Sixteen percent of patients in our study received NAC with partial or complete response facilitating breast conservation. ${ }^{38}$ The distant recurrence rate was $10 \%$ in our series reflecting a higher proportion of women with high-risk disease, emphasizing the safety of the breast conservation approach in this group.

The authors would like to emphasize that most women with high-risk disease would be offered chest wall radiotherapy even after mastectomy, which in turn could impact negatively on the esthetic outcome of immediate breast reconstruction. ${ }^{39}$ The impact of radiotherapy on PBR with lateral CWPF did not seem to impact the esthetic outcome significantly in our series. The perforator flaps appear sturdy with regards to their vascularity and volume maintenance. Only two patients required contralateral breast surgery to achieve symmetry, which was predominantly due to over-correction at the time of surgery.

In our experience, PBR techniques provide a valuable alternative to pursue the option of breast conservation. The data presented with a median follow-up of 4.5 years confirms the safety of the technique. The local recurrences observed were low, in keeping with published data on the safety of oncoplastic BCS. ${ }^{31}$ The distant recurrence rate observed was $10.5 \%$; the majority were triple negative cancers. This falls within the reported range of distant recurrences at $3.7 \%$ to $15.6 \%$ in various studies with a ollow-up between 3-5 years. ${ }^{31,40}$ A significantly high proportion of patients in our cohort were high-risk cancers; with $35 \%$ being triple negative or Her-2 positive and almost $50 \%$ patients had node-positive disease. The data supports consideration of mastectomy avoidance, where feasible, in high-risk disease, as distant recurrence dictates long-term outcome in such cases. The literature supports the equivalence of BCS and radiotherapy to mastectomy, ${ }^{30,41}$ and similar results have been shown in a metaanalysis for women under 40 years of age. $^{42}$ 


\section{Financial and Resource Implications}

These techniques provide means to achieve improved quality-of-ife for women with breast cancer without adding strain on the resources. These facilitate quicker recovery, short hospital stay, complications within acceptable limits compared to simple BCS and lower risks and reduced morbidity compared with mastectomy and reconstruction, enhanced psychosocial outcomes, and, finally, reduced need for maintenance or contralateral surgery in the future.

The authors acknowledge that the limitation of this study is that this is a single center experience, but the outcomes are generalizable given the sample size, long follow-up period and multimodality treatment that's routinely practiced for breast cancer management.

\section{Conclusion}

The lateral CWPF flaps have added an important tool to oncoplastic armamentarium to reduce mastectomy rates, particularly in women with small-to-moderate sized nonptotic breasts.

In our experience, the majority of patients undergoing PBR achieved good-to-excellent results, maintained over time. No total flap loss was encountered in our series. The low local recurrence rates and other oncological outcomes over a median follow-up of 4.5 years (DDFS, DFS) establishes the safety of approach for treatment of breast cancer including high-risk cancers.

\section{Strengths and Limitations of the Study}

1. PBR with LCWPF provides an option to extend indications for breast conservation surgery for women undergoing surgery for breast cancer and avoid mastectomy.

2. This approach results in excellent esthetic outcomes with improved psychological well-being of patients.

3. This study establishes the maintained esthetic outcome despite radiotherapy after a median follow-up of 54 months.

4. This study has shown a low local recurrence rate, even in high-risk cancers, after a median follow-up of 54 months, establishing the oncological safety of the procedure to promote breast conservation option with PBR.

5. This approach has potential applications in smokers in experienced hands, where alternative options could be met with higher complication rates.

6. The limitation of the study is lack of comparable data with other forms of breast conservation surgery.

\section{Acknowledgment}

The authors thank Mrs. Neerja Agrawal for her advice on statistics for this manuscript.

\section{Disclosure}

The authors report no conflicts of interest for this work.

\section{References}

1. Bertozzi N, Pesce M, Santi PL, Raposio E. Oncoplastic breast surgery: comprehensive review. Eur Rev Med Pharmacol Sci. 2017;21 (11):2572-2585.

2. Curigliano G, Burstein HJ, Winer EP, et al. De-escalating and escalating treatments for early-stage breast cancer: the St. Gallen international expert consensus conference on the primary therapy of early breast cancer 2017. Ann Oncol. 2017;28(8):1700-1712. doi:10.1093/ annonc $/ \mathrm{mdx} 308$

3. Waljee JF, Hu ES, Ubel PA, Smith DM, Newman LA, Alderman AK. Effect of esthetic outcome after breast-conserving surgery on psychosocial functioning and quality of life. J Clin Oncol. 2008;26 (20):3331-3337. doi:10.1200/JCO.2007.13.1375

4. Kim MK, Kim T, Moon HG, et al. Effect of cosmetic outcome on quality of life after breast cancer surgery. Eur J Surg Oncol. 2015;41 (3):426-432. doi:10.1016/j.ejso.2014.12.002

5. Thomas PR, Ford HT, Gazet JC. Use of silicone implants after wide local excision of the breast. Br J Surg. 1993;80(7):868-870. doi:10.1002/bjs. 1800800720

6. Holmstrom H, Lossing C. The lateral thoracodorsal flap in breast reconstruction. Plast Reconstr Surg. 1986;77(6):933-943. doi:10.1097/00006534-198606000-00010

7. Roy PG. One-stage vs. two-stage approach for partial breast reconstruction with lateral chest wall perforator flaps. Cancer Treat Res Commun. 2016;9:56-61. doi:10.1016/j.ctarc.2016.06.003

8. Youssif S, Hassan Y, Tohamy A, et al. Pedicled local flaps: a reliable reconstructive tool for partial breast defects. Gland Surg. 2019;8 (5):527-536. doi:10.21037/gs.2019.09.06

9. Ogawa T, Hanamura N, Yamashita M, Ri Y, Kuriyama N, Isaji S. Usefulness of breast-volume replacement using an inframammary adipofascial flap after breast-conservation therapy. Am $J$ Surg. 2007;193(4):514-518. doi:10.1016/j.amjsurg.2006.07.018

10. Kijima Y, Yoshinaka H, Hirata M, et al. Oncoplastic surgery combining partial mastectomy and immediate volume replacement using a thoracodorsal adipofascial cutaneous flap with a crescent-shaped dermis. Surg Today. 2014;44(11):2098-2105. doi:10.1007/s00595013-0812-1

11. Kijima Y, Yoshinaka H, Hirata M, et al. Immediate reconstruction using a modified thoracodorsal adipofascial cutaneous flap after partial mastectomy. Breast. 2011;20(5):464-467.

12. Takeda $\mathrm{M}$, Ishida $\mathrm{T}$, Ohnuki $\mathrm{K}$, et al. Breast conserving surgery with primary volume replacement using a lateral tissue flap. Breast Cancer. 2005;12(1):16-20. doi:10.2325/jbcs.12.16

13. Hamdi M, Craggs B, Stoel AM, Hendrickx B, Zeltzer A. Superior epigastric artery perforator flap: anatomy, clinical applications, and review of literature. $J$ Reconstr Microsurg. 2014;30(7):475-482. doi:10.1055/s-0034-1376399

14. Hamdi M, Van Landuyt K, de Frene B, Roche N, Blondeel P, Monstrey S. The versatility of the inter-costal artery perforator (ICAP) flaps. J Plast Reconstr Aesthet Surg. 2006;59(6):644-652. doi:10.1016/j.bjps.2006.01.006

15. Munhoz AM, Montag E, Arruda E, et al. Immediate conservative breast surgery reconstruction with perforator flaps: new challenges in the era of partial mastectomy reconstruction? Breast. 2011;20 (3):233-240. doi:10.1016/j.breast.2011.01.001 
16. Uppal RS, Stillaert FB, Hamdi M. Antiphospholipid syndrome-a rare cause of free flap thrombosis in perforator flap breast reconstruction. $J$ Plast Reconstr Aesthet Surg. 2008;61(3):347-348. doi:10.1016/j. bjps.2007.11.016

17. McCulley SJ, Schaverien MV, Tan VK, Macmillan RD. Lateral thoracic artery perforator (LTAP) flap in partial breast reconstruction. J Plast Reconstr Aesthet Surg. 2015;68(5):686-691. doi:10.1016/j.bjps.2015.01.008

18. Rusby JE, Paramanathan N, Laws SA, Rainsbury RM. Immediate latissimus dorsi miniflap volume replacement for partial mastectomy: use of intra-operative frozen sections to confirm negative margins. Am J Surg. 2008;196(4):512-518. doi:10.1016/j.amjsurg.2008.06.026

19. Zaha H. Partial breast reconstruction for the medial quadrants using the omental flap. Ann Surg Oncol. 2014;21(10):3358. doi:10.1245/ s10434-014-3907-z

20. McCulley SJ, Macmillan RD, Rasheed T. Transverse Upper Gracilis (TUG) flap for volume replacement in breast conserving surgery for medial breast tumours in small to medium sized breasts. $J$ Plast Reconstr Aesthet Surg. 2011;64(8):1056-1060. doi:10.1016/j. bjps.2011.03.016

21. Zaha H, Onomura M, Nomura H, Umekawa K, Oki M, Asato H. Free omental flap for partial breast reconstruction after breast-conserving surgery. Plast Reconstr Surg. 2012;129(3):583-587. doi:10.1097/ PRS.0b013e3182402cc6

22. Kim JB, Eom JR, Lee JW, Lee J, Park HY, Yang JD. Utility of two surgical techniques using a lateral intercostal artery perforator flap after breast-conserving surgery: a single-center retrospective study. Plast Reconstr Surg. 2019;143(3):477e-87e. doi:10.1097/ PRS.0000000000005374

23. Roy PG, Tenovici AA. Staged approach to partial breast reconstruction to avoid mastectomy in women with breast cancer. Gland Surg. 2017;6(4):336-342. doi:10.21037/gs.2017.03.08

24. O'Connell RL, DiMicco R, Khabra K, et al. Initial experience of the BREAST-Q breast-conserving therapy module. Breast Cancer Res Treat. 2016;160(1):79-89. doi:10.1007/s10549-016-3966-x

25. Goyal A, Dodwell D. POSNOC: a randomised trial looking at axillary treatment in women with one or two sentinel nodes with macrometastases. Clin Oncol. 2015;27(12):692-695. doi:10.1016/j. clon.2015.07.005

26. Hu JTA, Tenovici A, Parulekar V, Bhattacharyya M, Roy,PG. The impact of partial breast reconstruction with lateral chest wall perforator flaps on post-operative cancer surveillance. Ann Breast Surg. 2018;2(10):10. doi:10.21037/abs.2018.04.01

27. Hu J, Cuffolo G, Parulekar V, Chan V, Tenovici A, Roy PG. The results of surveillance imaging after breast Conservation surgery and partial breast reconstruction with chest wall perforator flaps; a qualitative analysis compared with standard breast-conserving surgery for breast cancer. Clin Breast Cancer. 2019;19(3):e422-e7. doi:10.1016/j.clbc.2019.01.010

28. Hopwood P, Fletcher I, Lee A, Al Ghazal S. A body image scale for use with cancer patients. Eur J Cancer. 2001;37(2):189-197. doi:10.1016/S0959-8049(00)00353-1

29. Pusic AL, Klassen AF, Scott AM, Klok JA, Cordeiro PG, Cano SJ. Development of a new patient-reported outcome measure for breast surgery: the BREAST-Q. Plast Reconstr Surg. 2009;124(2):345-353. doi:10.1097/PRS.0b013e3181aee807

Cancer Management and Research

\section{Publish your work in this journal}

Cancer Management and Research is an international, peer-reviewed open access journal focusing on cancer research and the optimal use of preventative and integrated treatment interventions to achieve improved outcomes, enhanced survival and quality of life for the cancer patient.
30. van Maaren MC, de Munck L, de Bock GH, et al. 10 year survival after breast-conserving surgery plus radiotherapy compared with mastectomy in early breast cancer in the Netherlands: a population-based study. Lancet Oncol. 2016;17(8):1158-1170. doi:10.1016/S1470-2045(16)30067-5

31. Clough KB, van la Parra RFD, Thygesen HH, et al. Long-term results after oncoplastic surgery for breast cancer: a 10-year Follow-up. Ann Surg. 2018;268(1):165-171. doi:10.1097/SLA.0000000000002255

32. Agrawal SK, Shakya SR, Nigam S, Sharma A, Datta SS, Ahmed R. Chest wall perforator flaps in partial breast reconstruction after breast conservation surgery: an additional oncoplastic surgical option. Ecancermedicalscience. 2020;14:1073. doi:10.3332/ecancer.2020.1073

33. Flanagan MR, Zabor EC, Romanoff A, et al. A comparison of patient-reported outcomes after breast-conserving surgery and mastectomy with implant breast reconstruction. Ann Surg Oncol. 2019;26 (10):3133-3140. doi:10.1245/s10434-019-07548-9

34. Hamdi M. Oncoplastic and reconstructive surgery of the breast. Breast. 2013;22(Suppl 2):S100-S105. doi:10.1016/j. breast.2013.07.019

35. Bartelink H, Maingon P, Poortmans P, et al. Whole-breast irradiation with or without a boost for patients treated with breast-conserving surgery for early breast cancer: 20-year follow-up of a randomised Phase 3 trial. Lancet Oncol. 2015;16(1):47-56. doi:10.1016/S14702045(14)71156-8

36. Soumian S, Parmeshwar R, Chandarana M, Marla S, Narayanan S, Shetty G. Chest wall perforator flaps for partial breast reconstruction: surgical outcomes from a multicenter study. Arch Plast Surg. 2020;47 (2):153-159. doi:10.5999/aps.2019.01186

37. Joshi S, Jaiswal D, Chougle Q, Wadasadawala T, Badwe RA. Transposition flap for the oncoplastic reconstruction of outer quadrant breast defects. J Plast Reconstr Aesthet Surg. 2020;74 (9):2176-2183. doi:10.1016/j.bjps.2020.12.037

38. Charfare H, Limongelli S, Purushotham AD. Neoadjuvant chemotherapy in breast cancer. $B r \quad J$ Surg. 2005;92(1):14-23. doi: $10.1002 /$ bjs. 4840

39. Rocco N, Catanuto G, Nava MB. Radiotherapy and breast reconstruction. Minerva Chir. 2018;73(3):322-328. doi:10.23736/ S0026-4733.18.07615-0

40. De La Cruz L, Blankenship SA, Chatterjee A, et al. Outcomes after oncoplastic breast-conserving surgery in breast cancer patients: a systematic literature review. Ann Surg Oncol. 2016;23 (10):3247-3258. doi:10.1245/s10434-016-5313-1

41. Litiere S, Werutsky G, Fentiman IS, et al. Breast conserving therapy versus mastectomy for stage I-II breast cancer: 20 year follow-up of the EORTC 10801 phase 3 randomised trial. Lancet Oncol. 2012;13 (4):412-419. doi:10.1016/S1470-2045(12)70042-6

42. Vila J, Gandini S, Gentilini O. Overall survival according to type of surgery in young $(\leq 40$ years) early breast cancer patients: a systematic meta-analysis comparing breast-conserving surgery versus mastectomy. Breast. 2015;24(3):175-181. doi:10.1016/j. breast.2015.02.002

The manuscript management system is completely online and includes a very quick and fair peer-review system, which is all easy to use. Visit http://www.dovepress.com/testimonials.php to read real quotes from published authors 